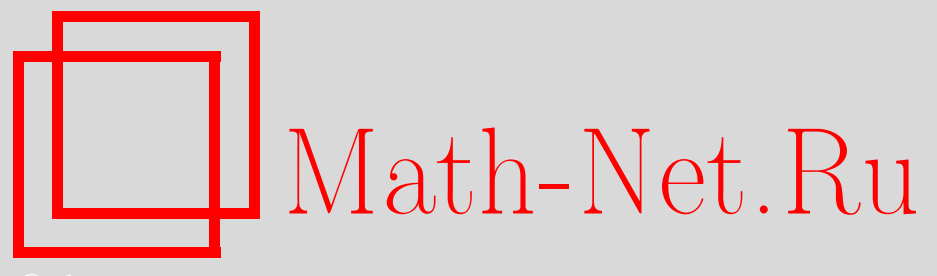

В. Н. Анисимов, В. Л. Литвинов, И. В. Корпен, Об одном методе получения аналитического решения волнового уравнения, описывающего колебания систем с движущимися границами, Вестн. Сам. гос. техн. ун-та. Сер. Физ.-мат. науки, 2012, выпуск 3(), 145151

DOI: https://doi.org/10.14498/vsgtu1079

Использование Общероссийского математического портала Math-Net.Ru подразумевает, что вы прочитали и согласны с пользовательским соглашением

http: //www . mathnet.ru/rus/agreement

Параметры загрузки:

IP: 52.6 .47 .48

26 апреля 2023 г., 17:20:46

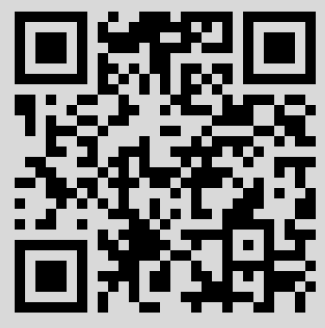


Вестн. Сам. гос. техн. ун-та. Сер. Физ.-мат. науки. 2012. № 3 (28). С. $145-151$

УДК 517.956.3:534.11

\title{
ОБ ОДНОМ МЕТОДЕ ПОЛУЧЕНИЯ АНАЛИТИЧЕСКОГО РЕШЕНИЯ ВОЛНОВОГО УРАВНЕНИЯ, ОПИСЫВАЮЩЕГО КОЛЕБАНИЯ СИСТЕМ С ДВИЖУЩИМИСЯ ГРАНИЦАМИ
}

\author{
В.Н. Анисимов, В.Л. Литвинов, И. В. Корпен \\ Сызранский филиал Самарского государственного технического университета, \\ 446001, Россия, Самарская обл., Сызрань, ул. Советская, 45. \\ E-mails: anisimov170159@mail.ru, vladlitvinov@rambler.ru
}

\begin{abstract}
Описан аналитический метод решения волнового уравнения с условиями, заданными на движущихся границах. C помощъю замены переменных в системе функциональных уравнений исходная краевая задача сведена к системе разностных уравнений с одним постоянным смещением, которая может быть решена с помощъю интегрального преобразования Лапласа. Получено выражение для амплитуды колебаний, соответствующих n-ной динамической моде в случае граничных условий первого рода. Данный метод позволяет рассмотреть более широкий класс граничных условий по сравнению с другими аналитическими методами решения краевых задач с движущимися границами.
\end{abstract}

Ключевые слова: волновое уравнение, колебания систем с двиюущимися границами, законь движения грании, амплитуда колебаний.

Одномерные системы с движущимися границами широко распространены в технике (канаты грузоподъемных установок [1], гибкие звенья передач [2] и т. д.). Наличие движущихся границ вызывает значительные затруднения при описании таких систем, поэтому здесь в основном используются приближенные методы решения [3,4]. Из аналитических методов наиболее эффективным является метод, предложенный в [5], который заключается в подборе новых переменных, останавливающих границы и сохраняющих инвариантность уравнения. В [6] решение ищется в виде суперпозиции двух волн, бегущих навстречу друг другу. В результате этого автору удалось решить волновое уравнение с граничными условиями первого рода, заданными на одной движущейся и одной неподвижной границах. Эффективен также метод, используемый в [7] и заключающийся в замене геометрической переменной на чисто мнимую, что позволяет свести волновое уравнение к уравнению Лапласа и применить для решения методику теории функций комплексного переменного.

Решения, полученные с помощью перечисленных методов, ограничены граничными условиями первого рода. $\mathrm{K}$ недостаткам методов относится также то, что в случае двух движущихся границ начальные условия, заданные при $t=0$, не могут быть учтены.

Перечисленных недостатков лишен развиваемый в данной статье метод решения таких задач, в котором сочетается методика, используемая в $[5,6]$.

Пусть движение системы описывается волновым уравнением

$$
U_{\tau \tau}(\xi, \tau)-U_{\xi \xi}(\xi, \tau)=0
$$

Валерий Николаевич Анисимов (к.ф.-м.н., доц.), доцент, каф. общетеоретических дисциплин. Владислав Лъвович Литвинов, преподаватель, каф. общетеоретических дисциплин. Инна Владимировна Корпен (к.п.н.), доцент, каф. общетеоретических дисциплин. 
при граничных условиях первого рода

$$
\begin{gathered}
U\left(l_{1}(\tau), \tau\right)=F_{1}(\tau), \quad l_{1}(0)=0 \\
U\left(l_{2}(\tau), \tau\right)=F_{2}(\tau), \quad l_{2}(0)=1, \quad l_{2}(\tau)>l_{1}(\tau)
\end{gathered}
$$

и начальных условиях

$$
U(\xi, 0)=\Phi_{0}(\xi), \quad U_{\tau}(\xi, 0)=\Phi_{1}(\xi)
$$

Здесь $\tau \geqslant 0$ - безразмерное время; $\xi \in[0,1]$ - безразмерная пространственная координата $\left(l_{1}(\tau) \leqslant \xi \leqslant l_{2}(\tau)\right) ; l_{1}(\tau), l_{2}(\tau)$ - законы движения границ; $\Phi_{0}(\xi), \Phi_{1}(\xi), F_{1}(\tau), F_{2}(\tau)$ - заданные функции, допускающие разрывы первого рода.

Для решения задачи используем представление Даламбера. Общее решение уравнения (1) имеет вид

$$
U(\xi, \tau)=g(\tau+\xi)+G(\tau-\xi)
$$

где $g(z)$ и $G(z)$ - произвольные функции, которые необходимо определить из начальных и граничных условий, $z$ - независимая переменная.

Подставляя решение (4) в граничные (2) и начальные (3) условия, получим

$$
\begin{gathered}
\left\{\begin{array}{l}
g\left(\tau+l_{1}(\tau)\right)+G\left(\tau-l_{1}(\tau)\right)=F_{1}(\tau), \\
g\left(\tau+l_{2}(\tau)\right)+G\left(\tau-l_{2}(\tau)\right)=F_{2}(\tau) ;
\end{array}\right. \\
\begin{cases}g(\xi)+G(-\xi)=\Phi_{0}(\xi), & 0 \leqslant \xi \leqslant 1, \\
g^{\prime}(\xi)+G^{\prime}(-\xi)=\Phi_{1}(\xi), & 0 \leqslant \xi \leqslant 1 .\end{cases}
\end{gathered}
$$

Из системы (6) найдём функции $g(\xi)$ и $G(\xi)$ :

$$
\begin{array}{ll}
g(\xi)=\frac{1}{2}\left[\Phi_{0}(\xi)+\int_{0}^{\xi} \Phi_{1}(\zeta) d \zeta\right], & 0 \leqslant \xi \leqslant 1 \\
G(\xi)=\frac{1}{2}\left[\Phi_{0}(-\xi)+\int_{0}^{\xi} \Phi_{1}(-\zeta) d \zeta\right], & -1 \leqslant \xi \leqslant 0 .
\end{array}
$$

В отличие от метода А. И. Весницкого [5], где в дифференциальное уравнение вводятся новые переменные, останавливающие границы и сохраняющие инвариантность уравнения, для упрощения задачи введём в систему (5) новые функции

$$
g(z)=r(\varphi(z)) ; \quad G(z)=R(\psi(z)) .
$$

Тогда система уравнений (5) примет вид

$$
\left\{\begin{array}{l}
r\left(\varphi\left(\tau+l_{1}(\tau)\right)\right)+R\left(\psi\left(\tau-l_{1}(\tau)\right)\right)=F_{1}(\tau) \\
r\left(\varphi\left(\tau+l_{2}(\tau)\right)\right)+R\left(\psi\left(\tau-l_{2}(\tau)\right)\right)=F_{2}(\tau)
\end{array}\right.
$$

Введём обозначения в первом уравнении системы (9):

$$
\varphi\left(\tau+l_{1}(\tau)\right)=z ; \quad \psi\left(\tau-l_{1}(\tau)\right)=z,
$$


а во втором уравнении этой системы-

$$
\varphi\left(\tau+l_{2}(\tau)\right)=z ; \quad \psi\left(\tau-l_{2}(\tau)\right)=z-1 .
$$

Другими словами, если функции $\varphi(z)$ и $\psi(z)$ удовлетворяют системе

$$
\left\{\begin{array}{l}
\varphi\left(\tau+l_{1}(\tau)\right)=\psi\left(\tau-l_{1}(\tau)\right) \\
\varphi\left(\tau+l_{2}(\tau)\right)=\psi\left(\tau-l_{2}(\tau)\right)+1
\end{array}\right.
$$

то система (5) принимает вид

$$
\left\{\begin{array}{l}
r(z)+R(z)=\theta_{1}(z) \\
r(z)+R(z-1)=\theta_{2}(z)
\end{array}\right.
$$

где $\theta_{1}(z)=F_{1}(0,5 \bar{\varphi}(z)+0,5 \bar{\psi}(z)) ; \theta_{2}(z)=F_{2}(0,5 \bar{\varphi}(z)+0,5 \bar{\psi}(z-1))$. Здесь $\bar{\varphi}(z)$, $\bar{\psi}(z)$ - функции, обратные к $\varphi(z)$ и $\psi(z)$.

Заметим, что из системы (10) функции $\varphi(z)$ и $\psi(z)$ определяются с точностью до константы в том смысле, что если $\varphi(z)$ и $\psi(z)$ - решение системы $(10)$, то $\varphi(z)+C$ и $\psi(z)+C$ также являются решением (здесь $C-$ произвольная постоянная). Поэтому для определённости можно выбрать такую функцию $\psi(z)$, что $\psi(-1)=-1$. При этом из второго уравнения системы $(10)$ при $\tau=0$ следует, что $\varphi(1)=0$. Из первого уравнения системы $(10)$ при $\tau=0$ получим $\varphi(0)=\psi(0)$.

С учётом замены (8) начальные условия (6) примут вид

$$
\begin{aligned}
& r(z)=g(\bar{\varphi}(z)), \quad \varphi(0) \leqslant z \leqslant 0 \\
& R(z)=G(\bar{\psi}(z)), \quad-1 \leqslant z \leqslant \psi(0),
\end{aligned}
$$

где функции $g(z)$ и $G(z)$ определяются выражениями (7).

Таким образом, начальная задача (1)-(3) сведена к системе разностных уравнений (11) с одним постоянным смещением при начальных условиях (12).

Из первого уравнения системы (11) получим

$$
R(z)=\theta_{1}(z)-r(z)
$$

После подстановки (13) во второе уравнение (11) будем иметь

$$
r(z)-r(z-1)=\theta(z),
$$

где $\theta(z)=\theta_{2}(z)-\theta_{1}(z-1)$. Для решения задачи $(14),(12)$ применим интегральное преобразование Лапласа:

$$
\bar{r}(p)=\frac{\bar{\theta}(p)}{1-e^{-p}}+e^{-p} \int_{-1}^{0} \frac{r(z) e^{-p z} d z}{\left(1-e^{-p}\right)},
$$

где $\bar{\theta}(p)$ - изображение функции $\theta(z)$. Оригинал данного изображения имеет вид

$$
r(z)=\int_{0}^{z} \theta(\zeta) \sum_{n=-\infty}^{\infty} e^{2 \pi n i(z-\zeta)} d \zeta+\sum_{n=-\infty}^{\infty} e^{2 \pi n i z} \int_{-1}^{0} r(\zeta) e^{-2 \pi n i \zeta} d \zeta
$$


Объединяя члены при положительных и отрицательных $n$, получим

$$
\begin{aligned}
r(z)=\int_{0}^{z} \theta(\zeta) d \zeta+2 \sum_{n=1}^{\infty} & \int_{0}^{z} \theta(\zeta) \cos [2 \pi n(z-\zeta)] d \zeta+ \\
& +\int_{-1}^{0} r(\zeta) d \zeta+2 \sum_{n=1}^{\infty} \int_{-1}^{0} r(\zeta) \cos [2 \pi n(z-\zeta)] d \zeta
\end{aligned}
$$

Рассмотрим свободные колебания системы $(\theta(z)=0)$. В этом случае из (4) с учётом (8), (13) и (15) следует, что

$$
U(\xi, \tau)=\sum_{n=1}^{\infty} V_{n}^{*}(\xi, \tau),
$$

где

$$
\begin{aligned}
& V_{n}^{*}(\xi, \tau)=\sin \{\pi n[\varphi(\tau+\xi)-\psi(\tau-\xi)]\} \times \\
& \times\left(A_{n}^{*} \cos \{\pi n[\varphi(\tau+\xi)+\psi(\tau-\xi)]\}-B_{n}^{*} \sin \{\pi n[\varphi(\tau+\xi)+\psi(\tau-\xi)]\}\right) ; \\
& A_{n}^{*}=4 \int_{-1}^{0} r(\zeta) \sin (2 \pi n \zeta) d \zeta ; \quad B_{n}^{*}=4 \int_{-1}^{0} r(\zeta) \cos (2 \pi n \zeta) d \zeta .
\end{aligned}
$$

Функцию $V_{n}^{*}(\xi, \tau)$ будем называть $n$-ным собственным колебанием системы, а функцию $\omega(\xi, \tau)=(\partial / \partial \tau)\{\pi n[\varphi(\tau+\xi)+\psi(\tau-\xi)]\}-$ мгновенной собственной частотой $n$-ного собственного колебания. Функция $\sin \{\pi n[\varphi(\tau+\xi)-\psi(\tau-\xi)]\}$ характеризует форму колебаний, и её принято называть $n$-ной динамической модой системы.

Рассмотрим теперь вынужденные колебания системы. При нулевых начальных условиях из (4) с учётом (8), (13) и (15) получим

$$
U(\xi, \tau)=\sum_{n=1}^{\infty} V_{n}(\xi, \tau)+D(\xi, \tau)
$$

где

$$
\begin{aligned}
& V_{n}(\xi, \tau)= 4 \sin \{\pi n[\varphi(\tau+\xi)-\psi(\tau-\xi)]\} \times \\
& \times\left(\cos \{\pi n[\varphi(\tau+\xi)+\psi(\tau-\xi)]\} \int_{0}^{\psi(\tau-\xi)} \theta(\zeta) \sin (2 \pi n \zeta) d \zeta-\right. \\
& \quad-4 \sin \{\pi n[\varphi(\tau+\xi)+\psi(\tau-\xi)]\} \int_{0}^{\psi(\tau-\xi)} \theta(\zeta) \cos (2 \pi n \zeta) d \zeta ; \\
& D(\xi, \tau)=\int_{\psi(\tau-\xi)}^{\varphi(\tau+\xi)} \theta(\zeta) d \zeta+\theta_{1}(\psi(\tau-\xi))+ \\
& \quad+2 \sum_{n=1}^{\infty} \int_{\psi(\tau-\xi)}^{\varphi(\tau+\xi)} \theta(\zeta) \cos \{2 \pi n[\varphi(\tau+\xi)-\zeta]\} d \zeta .
\end{aligned}
$$


Выражение (20) представляет собой ряд Фурье для функции

$$
D(\xi, \tau)= \begin{cases}\theta_{1}(\psi(\tau-\xi)), & \xi=l_{1}(\tau) \\ \theta(\varphi(\tau+\xi))+\theta_{1}(\psi(\tau-\xi)), & l_{1}(\tau)<\xi<l_{2}(\tau)\end{cases}
$$

При решении задач на резонансные свойства рассматриваются, главным образом, резонансные явления в механических объектах с движущимися границами, когда амплитуда колебаний во много раз превосходит амплитуду возмущающего воздействия. Поэтому в равенстве $(18)$ функцией $D(\xi, \tau)$ можно пренебречь как функцией одного порядка малости с функциями $F_{1}(\tau)$ и $F_{2}(\tau)$, характеризующими возмущающие воздействия.

Сравнивая выражения (16) и (18), (17) и (19), нетрудно заметить, что вынужденные колебания представляют собой суперпозицию собственных колебаний с изменяющимися во времени амплитудами:

$$
A_{n}=4 \int_{0}^{\psi(\tau-\xi)} \theta(\zeta) \sin (2 \pi n \zeta) d \zeta ; \quad B_{n}=4 \int_{0}^{\psi(\tau-\xi)} \theta(\zeta) \cos (2 \pi n \zeta) d \zeta
$$

Заметим, что из всех видов внешних воздействий наиболее распространенными являются гармонические нагрузки. Ограничимся рассмотрением случая, когда

$$
\theta(z)=B \cos W(z)
$$

где функция $W(z)$ класса $C^{1}$, а $B$ - константа, характеризующая интенсивность нагрузки. В этом случае равенства (21) можно переписать следующим образом:

$$
\begin{aligned}
& A_{n}=2 B\left[\int_{0}^{\psi(\tau-\xi)} \sin \Phi_{n 1}(\zeta) d \zeta+\int_{0}^{\psi(\tau-\xi)} \sin \Phi_{n 2}(\zeta) d \zeta\right] \\
& B_{n}=2 B\left[\int_{0}^{\psi(\tau-\xi)} \cos \Phi_{n 1}(\zeta) d \zeta+\int_{0}^{\psi(\tau-\xi)} \cos \Phi_{n 2}(\zeta) d \zeta\right]
\end{aligned}
$$

где $\Phi_{n 1}(\zeta)=2 \pi n \zeta-W(\zeta) ; \Phi_{n 2}(\zeta)=2 \pi n \zeta+W(\zeta)$.

Так как функции $2 \pi n \zeta$ и $W(\zeta)$ монотонно возрастают, фаза $\Phi_{n 2}(\zeta)$ быстро изменяется, что приводит к осциллированию с небольшой амплитудой соответствующих интегралов. Фаза же $\Phi_{n 1}(\zeta)$ может изменяться очень медленно. При этом наблюдается резонансное явление, которое характеризуется ростом интегралов, содержащих фазу $\Phi_{n 1}(\zeta)$. Из изложенного следует, что при возникновении резонанса рост амплитуды связан с возрастанием интегралов с фазой $\Phi_{n 1}(\zeta)$, интегралами же с фазой $\Phi_{n 2}(\zeta)$ можно пренебречь. Тогда полная амплитуда, определяемая по формуле $A_{n}^{2}(\tau)=A_{n}^{2}+B_{n}^{2}$, в точке $\xi=\xi_{0}(\tau)$, соответствующей максимальному размаху колебаний, будет иметь вид

$$
A_{n}^{2}(\tau)=4 B^{2}\left\{\left[\int_{0}^{b(\tau)} \cos \Phi_{n}(\zeta) d \zeta\right]^{2}+\left[\int_{0}^{b(\tau)} \sin \Phi_{n}(\zeta) d \zeta\right]^{2}\right\}
$$

где $b(\tau)=\psi\left(\tau-\xi_{0}(\tau)\right), \Phi_{n}(\zeta)=2 \pi n \zeta-W(\zeta)$. 
Возможность решения задачи (1)-(3) зависит от степени сложности граничных условий, а также возможности решения системы (10). Для решения таких систем в [5] использован обратный метод: по заданным $\varphi(z)$ и $\psi(z)$ из получающейся системы уравнений находятся законы движения границ $l_{1}(\tau)$ и $l_{2}(\tau)$. При задании функций $\varphi(z)$ и $\psi(z)$ в них вводится несколько произвольных постоянных. Зависимость найденных законов движения $l_{1}(\tau)$ и $l_{2}(\tau)$ от величин этих констант позволяет аппроксимировать достаточно разнообразные законы движения границ законами, полученными из решения обратной задачи.

Совокупность обратных решений достаточно широка [8]. Для примера приведём два таких решения:

1) для функций $\varphi(z)=B\left(e^{-\alpha z}-1\right)-C\left(e^{-\alpha}-1\right)-1, B=C+\left(e^{-\alpha}-1\right)^{-1}$; $\psi(z)=C\left(e^{\alpha z}-1\right)-C\left(e^{-\alpha}-1\right)-1$ из системы $(10)$ находятся следующие законы движения границ: $l_{1}(\tau)=\alpha^{-1} \ln \left[\left(B e^{-\alpha \tau}-C e^{\alpha \tau}\right) /(B-C)\right]$; $l_{2}(\tau)=1+l_{1}(\tau)$; здесь $\alpha, B, C, v$-постоянные величины;

2) для функций

$$
\varphi(z)=\psi(z)=\frac{\operatorname{Ln}[(v z+1) /(1-v)]}{\operatorname{Ln}[(1+v) /(1-v)]}-1
$$

находятся следующие законы движения границ: $l_{1}(\tau)=0 ; l_{2}(\tau)=v \tau+1$.

Первое из решений может быть использовано при изучении колебаний гибких звеньев передач [2]. Второе - при изучении колебаний канатов грузоподъемных установок при равномерном подъёме (спуске).

В заключение отметим, что приведённая в настоящей работе методика позволяет получить выражение для амплитуды колебаний, соответствующих $n$-ной динамической моде в случае граничных условий первого рода. Кроме того, описанный выше метод позволяет также рассмотреть следующий класс краевых условий [8]:

$$
\begin{array}{cc}
U_{\tau}\left(l_{1}(\tau), \tau\right)=F_{1}(\tau), & U_{\xi}\left(l_{2}(\tau), \tau\right)=F_{2}(\tau) \\
U\left(l_{1}(\tau), \tau\right)=F_{1}(\tau), & U_{\xi}\left(l_{2}(\tau), \tau\right)=F_{2}(\tau) \\
U\left(l_{1}(\tau), \tau\right)=F_{1}(\tau), & U_{\tau}\left(l_{2}(\tau), \tau\right)=F_{2}(\tau) .
\end{array}
$$

Насколько известно, решения волнового уравнения при граничных условиях (22) другими известными аналитическими методами получить крайне сложно.

\section{БИБЛИОГРАФИЧЕСКИЙ СПИСОК}

1. Савин Г.Н., Горошко О.А. Динамика нити переменной длины: Киев, 1962. 332 с. [Savin G. N. Goroshko O. A. Dynamics of variable length strands: Kiev, 1962. 332 pp.]

2. Самарин Ю.П., Анисимов В.Н. Вынужденные поперечные колебания гибкого звена при разгоне// Изв. вузов. Машиностроение, 1986. №12. С. 17-21. [Samarin Yu.P., Anisimov V. N. Forced transverse vibrations of the flexible link at dispersal// Izv. Vuzov. Mashinostroenie, 1986. no. 12. Pp. 17-21].

3. Анисимов В. Н., Литвинов В. Л. Исследование резонансных свойств механических объектов с движущимися границами при помощи метода Канторовича-Галёркина // Becmн. Сам. гос. техн. ун-та. Сер. Физ.-мат. науки, 2009. № 1(18). С. 149-158. [Anisimov V. N., Litvinov $V$. L. Investigation of resonance characteristics of mechanical objects with moving borders by application of the Kantorovich-Galyorkin method// Vestn. Samar. Gos. Tekhn. Univ. Ser. Fiz.-Mat. Nauki, 2009. no. 1(18). Pp. 149-158]. 
4. Горошко О.А., Савин Г.Н. Введение в механику деформируемых одномерных тел переменной длины. Киев: Наукова думка, 1971. 270 с. [Goroshko O. A., Savin G. N. Introduction in mechanics of one dimensional deformable bodies of variable length. Kiev: Naukova Dumka, 1971. 270 pp.]

5. Весницкий А. И. Волны в системах с движущимися границами и нагрузками. М.: Физматлит, 2001. 320 с. [Vesnitskii A. I. Waves in systems with moving boundaries and loads. Fizmatlit: Moscow, 2001. 320 pp.]

6. Весницкий А. И. Обратная задача для одномерного резонатора изменяющего во времени свои размеры // Изв. вузов. Радиофизика, 1538-1542. № 10; англ. пер.: Vesnitskii A. I. The inverse problem for a one-dimensional resonator the dimensions of which vary with time // Radiophys. Quantum Electronics, 1971. Vol.14, no. 10. Pp. 1209-1215.

7. Барсуков K.A., Григорян Г.А. К теории волновода с подвижными границами // Изв.вузов. Радиобизика, 1976. №2. С. 280-285; англ. пер.: Barsukov K. A., Grigoryan G.A. Theory of a waveguide with moving boundaries // Radiophys. Quantum Electronics, 1976. Vol. 19, no. 2. Pp. 194-200.

8. Анисимов В.Н., Литвинов В. Л. Резонансные свойства механических объектов с движущимися границами. Самара: СамГТУ, 2009. 131 с. [Anisimov V. N., Litvinov V. L. The resonance properties of mechanical objects with moving boundaries. Samara: SamGTU, 2009. 131 pp.]

Поступила в редакцию 10/V/2012;

в окончательном варианте - 25/VII/2012.

MSC: 35L05; 35L04, 35Q70

\title{
ON A METHOD OF ANALYTICAL SOLUTION OF WAVE EQUATION DESCRIBING THE OSCILLATIONS SISTEM WITH MOVING BOUNDARIES
}

\author{
V. N. Anisimov, V.L. Litvinov, I. V. Korpen \\ Syzran' Branch of Samara State Technical University, \\ 45, Sovetskaya str., Syzran', Samara region, 446001, Russia. \\ E-mails: anisimov170159@mail.ru, vladlitvinov@rambler.ru
}

The method of analytical solution of wave equation with the conditions, assigned on the moving boundaries, is described. With the aid of the change of variables in the system of functional equations the original boundary-value problem is brought to the system of difference equations with one fixed bias, which can be solved using the Laplace integral transform. The expression for amplitude of oscillation corresponding to $n$-th dynamic mode is obtained for the first kind boundary conditions. This method makes it possible to examine the broader class of boundary conditions in comparison with other exact methods of solving the boundary-value problems with the moving boundaries.

Key words: wave equation, variations of systems with moving boundaries, laws of boundary moving, amplitude of oscillation.

Original article submitted $10 / \mathrm{V} / 2012$; revision submitted 25/VII/2012.

Valeriy N. Anisimov (Ph. D. (Phys. \& Math.)), Associate Professor, Dept. of GeneralTheoretical Disciplines. Vladislav L. Litvinov, Teacher, Dept. of General-Theoretical Disciplines. Inna V. Korpen (Ph. D. (Educat.)), Associate Professor, Dept. of General-Theoretical Disciplines. 